\title{
A diagnostic dilemma of acute abdomen in pregnancy: Leiomyoma of the small intestine
}

\author{
Hüseyin Cengiz, Şükrü Yıldız, Cihan Kaya, Murat Ekin \\ Department of Obstetrics and Gynecology, Bakırköy Dr. Sadi Konuk Teaching and Research Hospital, Istanbul, Turkey
}

\begin{abstract}
Small intestinal tumours are rare and difficult to diagnose. This neoplasms may be responsible for haemorrhage, occlusion, perforation and subsequent emergent surgeries. A 28 year old G2P1 woman in her 22nd week of pregnancy was referred to our emergency department with a complaint of left lower abdominal pain that had begun the day before. She underwent an emergent laparotomy with the general surgeons. Histopathological examination defined the diagnosis of leiomyoma of the small intestine. Gastrointestinal pathologies should always be taken into consideration in the differential diagnosis of acute abdomen in pregnancy. (J Turk Ger Gynecol Assoc 2014; 15: 60-2)
\end{abstract}

Key words: Acute abdomen, pregnancy, small intestinal tumour

Received: 23 July, $2013 \quad$ Accepted: 07 August, $2013 \quad$ Available Online Date: 30 January, 2014

\section{Introduction}

Small intestinal tumours are rare and difficult to diagnose. These neoplasms may cause haemorrhage, occlusion, and perforation, and therefore, subsequent emergent surgeries may have to be performed (1). We report a case of a pregnant woman who underwent surgery for suspected ovarian torsion during the second trimester of pregnancy and was subsequently diagnosed as having leiomyoma of the small intestine. To the best of our knowledge, this is the first reported case of leiomyoma of the small intestine causing acute abdomen during pregnancy in the English literature.

\section{Case Presentation}

A 28-year-old woman (gravida 2 para 1) who was 22 weeks pregnant was referred to our emergency department with a complaint of left lower abdominal pain that she had been experiencing since the previous day. She presented with severe nausea and vomiting but did not report vaginal discharge, constipation, or rectal bleeding. Physical examination showed that she had a gravid abdomen corresponding to 20-22 weeks of pregnancy and tenderness, with guarding in her left lower abdomen. Abdominopelvic colour Doppler sonography revealed a solid mass, measuring $8 \times 10 \mathrm{~cm}$, with no vascularisation in the left pelvic region and a living foetus of 22 weeks gestation. The patient experienced progressively worsening pain and vomiting. She underwent emergent laparotomy because of the possibility of torsion due to adnexal pathology. During abdominal exploration, the ovaries and tubes appeared normal and the uterus appeared enlarged because of pregnancy. A semi-solid, dark brownish-red, infarcted mass, measuring $8 \times 10 \mathrm{~cm}$, was observed originating from the jejunum (Figure 1). The mass was resected with a $10 \mathrm{~cm}$ segment of the jejunum, and end-to-end anastomosis was performed in the usual manner by general surgeons. The final histopathological diagnosis was leiomyoma of the small intestine (Figure 2). The postoperative course of the patient was uneventful, and she was discharged home on the seventh postoperative day. Thereafter, she had an uncomplicated pregnancy and delivered a full-term baby.

\section{Discussion}

The incidence of adnexal masses during pregnancy has been reported to be $1-2 \%$. These pelvic masses are usually defined as functionally or hormonally active cysts that resolve by the $16^{\text {th }}$ gestational week. The masses that have been reported are mature cystic teratomas and cystadenomas (2). The major concern regarding these masses is the possibility of complications such as torsion, rupture, and malignancy (3).

On the other hand, pelvic masses could be attributed to extragonadal causes as well. Small bowel neoplasms such as leiomyoma, lipoma, hamartoma, or desmoid tumours are benign and usually asymptomatic (4). Leiomyomas are the most common type of small intestinal tumours, with an incidence of $20-30 \%$. The tumour is most com- 


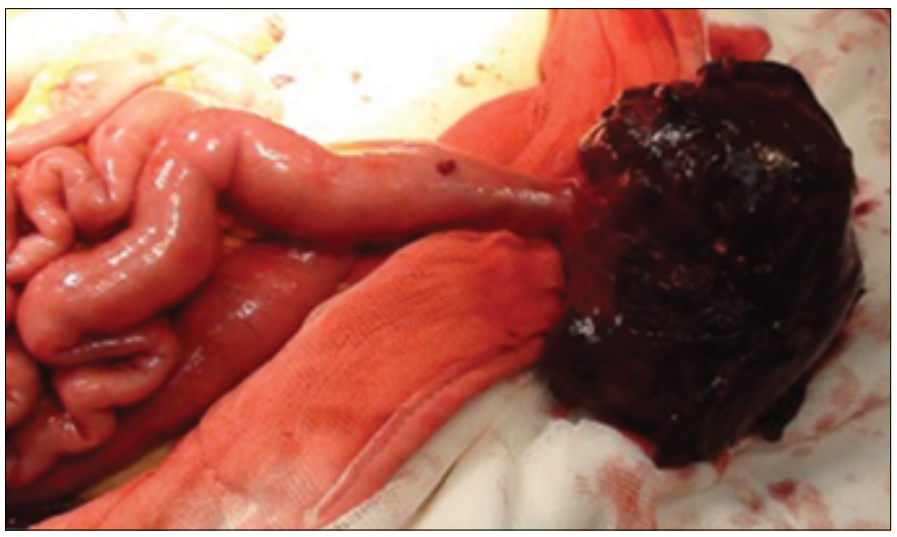

Figure 1. A semi-solid dark brownish-red mass originating from the small intestine

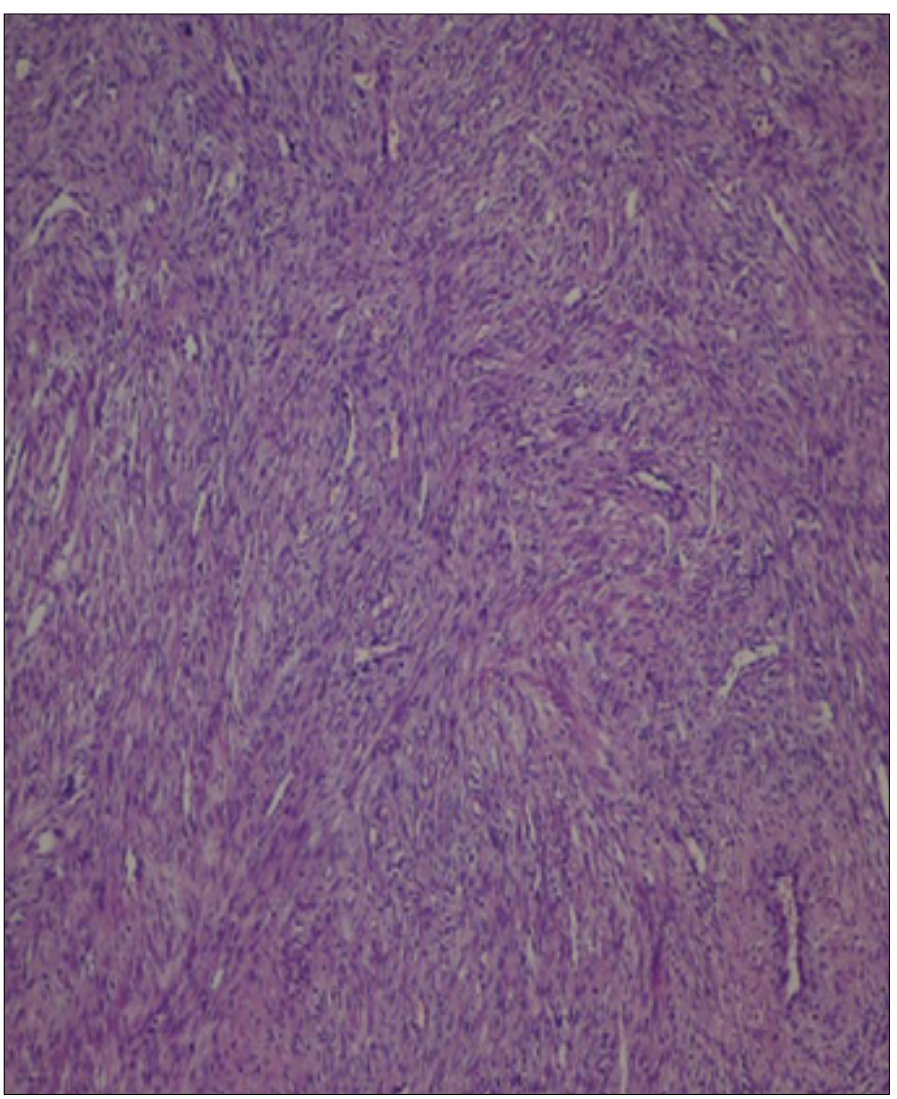

Figure 2. Histopathological appearance of leiomyoma with fusiformshaped, interlacing bundles of smooth-muscle cells with no necrosis or mitotic activity

monly located in the stomach, but rarely in the jejunum, ileum, or duodenum. Leiomyomas present with 4 different growth patterns: intraluminal, intramural, extraluminal, and dumbbell-shaped (5). The presenting symptoms depend on the location of the tumours. The most common presenting symptoms are intermittent gastrointestinal bleeding, obstruction, and intestinal invagination (5). Although most leiomyomas remain asymptomatic and are diagnosed incidentally during laparotomy, iron deficiency anaemia and obstructive symptoms are important in alerting one to the presence of leiomyomas (5). However, the diagnosis of small intestinal neoplasms is often delayed because the small intestine is relatively difficult to access during routine endoscopy. Magnetic resonance imaging (MRI) is generally considered safe during pregnancy (6). Moreover, it can also be used to determine the extent of possible malignancy and aid in the diagnosis of acute bowel conditions such as appendicitis and inflammatory bowel disease (7). In the case of our patient, MRI could have been useful in the differential diagnosis; however, this imaging modality is not available in emergency conditions.

The most significant problem in the diagnosis of the present case was the ongoing pregnancy and the absence of a history suggestive of intestinal pathologies. In the present case, the surgeons examined the patient preoperatively but were unable to make a diagnosis. However, we operated on the patient together with general surgeons. The tumour was resected, and end-to-end anastomosis was performed. General surgeons should participate in surgeries where the diagnosis is challenging and the patient is pregnant.

In the literature, there are reports on cases of intestinal pathologies such as gastrointestinal stromal tumours (GISTs) presenting during pregnancy (8). Leiomyomas usually mimic GISTs and GISTs mostly mimic ovarian malignancies and uterine leiomyomas on ultrasonography (9). However, histopathologically, leiomyomas are benign tumours that are negative for CD117 and CD34 and positive for smooth muscle actin and desmin. Furthermore, mitosis is the most important criterion for discrimination of these tumours (10). Distinction of the 2 tumours is important because leiomyomas, but not GISTs, can be treated with a simple enucleation.

To the best of our knowledge, this is the first case report describing a leiomyoma of the proximal jejunum that mimicked other gynaecologic masses in a pregnant woman who presented with acute abdomen due to infarction of the mass. The diagnosis was complicated because the symptoms were nonspecific and were obscured by the ongoing pregnancy. Moreover, it is difficult to take a decision regarding surgery in such cases considering the high rates of maternal complications, foetal loss, and premature births.

\section{Ethics Committee Approval: N/A.}

Informed Consent: Written informed consent was obtained from patient who participated in this case.

Peer-review: Externally peer-reviewed.

Author contributions: Concept -SS.Y.; Design -Ş.Y.; Supervision M.E.; Resource-H.C., S.Y., C.K.; Materials - H.C., S.Y., C.K.; Data Collection\&/or Processing - C.K.; Analysis\&/or Interpretation C.K.; Literature Search - H.C.; Writing - H.C.

Conflict of Interest: No conflict of interest was declared by the authors.

Financial Disclosure: The authors declared that this study received no financial support. 


\section{References}

1. Bölükbasi H, Nazli $\mathrm{O}$, Tansug $\mathrm{T}$, Bozdag $\mathrm{AD}$, Isgiider AS, Yaman I, et al. Gastrointestinal stromal tumors (GISTs): analysis of 20 cases. Hepatogastroenterology 2006; 53: 385-8.

2. Ergenoglu AM, Yeniel AO, Mermer T. Adnexal masses in pregnancy: clinical approach and pathological findings. Ege Journal of Medicine 2010; 49: 37-40

3. Mathevet P, Nessah K, Dargent D, Mellier G. Laparoscopic management of adnexal masses in pregnancy: a case series. Eur J Obstet Gynecol Reprod Biol 2003; 108: 217-22. [CrossRef]

4. Coco C, Rizzo G, Manno A, Mattana C, Verbo A. Surgical treatment of small bowel neoplasms Eur Rev Med Pharmacol Sci 2010; 14: 327-33.

5. Sunamak O, Karabicak I, Aydemir I, Aydogan F, Guler E, Cetinkaya $\mathrm{S}$, et al. An intraluminal leiomyoma of the small intestine causing invagination and obstruction: a case report. Mt Sinai J Med 2006; 73: 1079-81.

6. Hoover K, Jenkins TR. Evaluation and management of adnexal mass in pregnancy. Am J Obstet Gynecol 2011; 205: 97-102. [CrossRef]

7. Glanc P, Salem S, Farine D. Adnexal masses in pregnant patient: a diagnostic and management challenge. Ultrasound Q 2008; 24: 225-40. [CrossRef]

8. Scherjon S, Lam WF, Gelderblom H, Jansen FW. Gastrointestinal stromal tumor in pregnancy: a case report. Case Rep Med 2009; 2009: 456402.

9. Pinto V, Ingravallo G, Cicinelli E, Pintucci A, Sambati GS, Marinaccio $\mathrm{M}$, et al. Gastrointestinal stromal tumors mimicking gynecological masses on ultrasound: a report of two cases. Ultrasound Obstet Gynecol 2007; 30: 359-361 [CrossRef]

10. Abraham SC. Distinguishing gastrointestinal stromal tumors from their mimics: an update. 